Pacific

Journal of

Mathematics

\title{
MODULI SPACES FOR BONDAL QUIVERS
}

AARON BERGMAN AND Nicholas J. PROUdFOOT 


\title{
MODULI SPACES FOR BONDAL QUIVERS
}

\author{
Aaron Bergman ANd Nicholas J. Proudfoot
}

\begin{abstract}
Given a sufficiently nice collection of sheaves on an algebraic variety $V$, Bondal explained how to build a quiver $Q$ along with an ideal of relations in the path algebra of $Q$ such that the derived category of representations of $Q$ subject to these relations is equivalent to the derived category of coherent sheaves on $V$. We consider the case in which these sheaves are all locally free and study the moduli spaces of semistable representations of our quiver with relations for various stability conditions. We show that $V$ can often be recovered as a connected component of such a moduli space, and we describe the line bundle induced by a GIT construction of the moduli space in terms of the input data. In certain special cases, we interpret our results in the language of topological string theory.
\end{abstract}

An algebraic variety $V$ is completely determined by the abelian category $\operatorname{Coh}(V)$ of coherent sheaves on $V$ [Gabriel 1962], and it is therefore a natural problem to find a way to describe this category in concrete terms. If $V$ is affine, then $\operatorname{Coh}(V)$ is nothing more than the category of finitely generated modules over the algebra of global functions on $V$. If we have a presentation of this algebra, this may be interpreted as a "presentation" of the category $\operatorname{Coh}(V)$. In the projective case, it is unreasonable to expect $\operatorname{Coh}(V)$ to be equivalent to the category of modules over any ring. It is sometimes the case, however, that such an equivalence can be constructed after passing to the bounded derived category $\mathscr{D}^{b} \operatorname{Coh}(V)$. The derived category carries less information than the abelian category $\operatorname{Coh}(V)$, but it is enough to reconstruct such invariants as cohomology, K-theory, and higher Chow groups, as well as a great deal of information about the birational geometry of $V$. If $V$ is Calabi-Yau, then an object of $\mathscr{D}^{b} \operatorname{Coh}(V)$ may be thought of as a D-brane in type IIB topological string theory on $V$ [Aspinwall and Donagi 1998; Douglas 2001; Sharpe 1999]. This category is therefore of significant physical interest, and

MSC2000: 18E30, 16G20, 14L24.

Keywords: quiver, exceptional collection, D-brane.

Bergman is supported by the National Science Foundation under grant numbers PHY-0071512 and PHY-0455649, and the US Navy, Office of Naval Research, grant numbers N00014-03-1-0639 and N00014-04-1-0336, Quantum Optics Initiative. Proudfoot is supported by a National Science Foundation Postdoctoral Research Fellowship. 
is a fundamental ingredient in the formulation of homological mirror symmetry [Kontsevich 1995].

Let us describe more concretely how one might attempt to construct such an equivalence. Given an object $E$ of $\operatorname{Coh}(V)$, there is a natural functor $F$ from $\operatorname{Coh}(V)$ to the category of finitely generated right modules over the endomorphism algebra $\operatorname{End}(E)$, or left modules over the opposite algebra $\operatorname{End}(E)^{\mathrm{op}}$, taking a sheaf $\mathscr{F}$ to the module $\operatorname{Hom}(E, \mathscr{F})$. This functor will almost never be either faithful or essentially surjective, but if $E$ satisfies certain technical conditions, then Rickard shows that the right derived functor $R F$ from $\mathscr{D}^{b} \operatorname{Coh}(V)$ to the bounded derived category of left modules over $\operatorname{End}(E)^{\text {op }}$ will be an equivalence. (See Definition 1.2 and Theorem 1.3 for more details.) If $E$ decomposes as a direct sum of smaller objects $E=\bigoplus_{i=1}^{n} E_{i}$, then $\operatorname{End}(E)^{\mathrm{op}}$ may be expressed as the path algebra of a quiver with $n$ nodes, modulo certain relations (which may not be admissible). One should think of the description of such a quiver along with its relations as an analogue of a presentation of the coordinate ring of an affine variety.

Much work has gone into finding such collections of sheaves on projective varieties. The goal of this paper is not to find these collections, but rather to assume that one is given, and to study various moduli spaces of representations of the corresponding algebra. If the sheaves in the collection are vector bundles, there is a tautological map from $V$ to the moduli stack of quiver representations, taking a point $p$ to $F\left(\mathscr{O}_{p}\right)$, a representation in which the vector space associated to the node $i$ is equal to the dual of the fiber of $E_{i}$ at $p$. Thus, we restrict to representations in which the dimension of the vector space at node $i$ is the rank of the vector bundle $E_{i}$. Our goal is to consider coarse moduli spaces of semistable representations for various choices of stability condition and to relate these spaces to $V$. The representation stack may be presented as the quotient of an affine variety by the action of an algebraic group $G$, so these moduli spaces can be constructed as geometric invariant theory (GIT) quotients with respect to some character $\chi$ of $G$. In general, $V$ need not map to such a space as the representations in the image of the tautological map may not be semistable. Even if $V$ does map to one of these moduli spaces, the map may not be an inclusion, as representations whose closures in the stack intersect in a semistable representation are identified in the moduli space. In Section 2, the main section of this paper, we address the problem of determining when this map exists, and when it does, we study its structure. The GIT construction gives us not just a moduli space, but a moduli space equipped with an ample line bundle. Under suitable hypotheses, we show that $V$ may be identified with (a connected component of) the moduli space of stable quiver representations, and we identify the induced line bundle on $V$ in terms of $\chi$ and the vector bundles with which we started (Theorem 2.4).

Section 3 is devoted to a case of physical interest, in which $V$ is the total space 
of the dual of an ample line bundle $L$ on a projective variety $X$, and the collection on $V$ is pulled back from a particularly nice collection of line bundles on the base. In this case we study the affine quotient $M_{0}$ and prove that it has an irreducible component whose canonical reduced subvariety is isomorphic to $V_{0}$, the affine variety obtained from $V$ by collapsing the zero section of the bundle. If $L$ is the anticanonical bundle on a Fano survace, this result may be interpreted in the language of topological string theory as in the physics paper [Bergman and Proudfoot 2006]. The quiver moduli space $M_{0}$ parameterizes ground states of a quantum field theory that describes the behavior of open strings ending on a certain D-brane supported at the tip of the Calabi-Yau cone $V_{0}$. In general, the quantum field theory associated to a D-brane contains fields that are sections of the normal cone to the support of the D-brane. In this case, the normal cone is $V_{0}$ itself, and a section is simply a point in $V_{0}$. For physical reasons, it is expected that the space of sections of this normal cone should be a component of the moduli space of vacua in the quantum field theory, and therefore that $V_{0}$ should be a component of the quiver moduli space. Up to the issue of reducedness of $M_{0}$, this is now a theorem.

A special case of the situation discussed above occurs when the collection on $X$ is a simple helix (see Example 1.12). In Section 4, we construct the Fano variety $X$ with its anticanonical line bundle as a GIT quotient of a smooth variety with respect to a canonical polarization (Theorem 4.3). In particular, we obtain a result along the lines of those of Section 2 while eliminating the dependence on the choice of character $\chi$.

\section{Bondal quivers}

Let $Q$ be a directed graph with finitely many nodes $\{1, \ldots, n\}$, and let $\mathfrak{k}$ be an algebraically closed field. Let $P_{i j}(Q)$ denote the $\mathfrak{k}$-vector space spanned by the set of all paths in $Q$ from the node $i$ to the node $j$, including the path of length zero at each vertex. The direct sum $P(Q)=\bigoplus P_{i j}(Q)$ is naturally an algebra over $\mathfrak{k}$ with multiplication $P_{j k} \otimes P_{i j} \rightarrow P_{i k}$ given by concatenation of paths. Let $I \subseteq P(Q)$ be a two-sided homogeneous ideal contained in the square of the ideal of paths of nonzero length; such an ideal is called admissible. The pair $\mathbf{Q}=(Q, I)$ with $I$ admissible is called a quiver with relations. The algebra $P(\mathbf{Q}):=P(Q) / I$ is called the path algebra of $\mathbf{Q}$ and inherits a grading $P(\mathbf{Q})=\bigoplus P_{i j}(\mathbf{Q})$.

To any quiver with relations $\mathbf{Q}$, we may associate a $\mathfrak{k}$-linear category $\mathscr{C}(\mathbf{Q})$ with objects $\{1, \ldots, n\}$, and morphisms from $i$ to $j$ equal to $P_{i j}(\mathbf{Q})$. A representation of $\mathbf{Q}$ is defined to be a functor of $\mathfrak{k}$-linear categories from $\mathscr{C}(\mathbf{Q})$ to the category Vect $_{\mathfrak{k}}$ of $\mathfrak{k}$-vector spaces. Equivalently, it is a left module over the path algebra $P(\mathbf{Q})$. Let $\operatorname{Rep}(\mathbf{Q})$ denote the abelian category of representations of $\mathbf{Q}$. 
Let $\mathscr{C}$ be a $\mathfrak{k}$-linear abelian category and consider a finite collection $E_{1}, \ldots, E_{n}$ of objects in $\mathscr{C}$. The algebra $A:=\operatorname{End}_{\mathscr{C}}\left(\oplus E_{i}\right)^{\text {op }}$ has a distinguished collection $\left\{e_{i}\right\}$ of idempotents, where $e_{i}$ acts as $\delta_{i j}$ times the identity endomorphism on $E_{j}$. Suppose that $A$ is equipped with a grading by the natural numbers, with each graded piece finite-dimensional, and that the degree zero part $A_{0}$ is spanned by the idempotents $\left\{e_{i}\right\}$. It then makes sense to define the one-dimensional representation

$$
S_{i}:=A / A_{+}+\mathfrak{k}\left\{e_{j} \mid j \neq i\right\}
$$

on which $e_{i}$ acts as the identity, and all other idempotents and all elements of positive degree act by zero. Let $Q$ be a quiver on $n$ vertices with arrows from $i$ to $j$ given by a basis for $\operatorname{Ext}_{A}^{1}\left(S_{i}, S_{j}\right)^{\vee}$. There is a map

$$
\operatorname{Ext}_{A}^{2}\left(\oplus S_{i}, \oplus S_{i}\right)^{\vee} \rightarrow \bigoplus_{k \geq 2}\left(\operatorname{Ext}_{A}^{1}\left(\oplus S_{i}, \oplus S_{i}\right)^{\vee}\right)^{\otimes k}
$$

given by the $A_{\infty}$ structure on $\operatorname{Ext}_{A}^{\bullet}\left(\oplus S_{i}, \oplus S_{i}\right)$, whose image generates an admissible ideal $I \subseteq P(Q)$. Let $\mathbf{Q}$ be the corresponding quiver with relations; we refer to $\mathbf{Q}$ as the Bondal quiver for the collection $E_{1}, \ldots, E_{n}$. The following proposition may have been known to the experts for some time, but the first proof of it of which we are aware has recently been given by Segal [2007, 2.13].

Proposition 1.1. The path algebra $P(\mathbf{Q})$ is isomorphic to $A$.

There is a natural functor $F: \mathscr{C} \rightarrow \operatorname{Rep}(\mathbf{Q})$ taking an object $\mathscr{F} \in \mathscr{C}$ to a representation of $\mathbf{Q}$ in which the node $i$ is mapped to the vector space $\operatorname{Hom}_{\mathscr{C}}\left(E_{i}, \mathscr{F}_{F}\right)$. This functor is left exact and thus (provided that there exists a nice class of complexes adapted to $F$ ) induces a right-derived functor $R F: \mathscr{D}(\mathscr{C}) \rightarrow \mathscr{D} \operatorname{Rep}(\mathbf{Q})$ on unbounded derived categories.

Definition 1.2. An object $E$ of $\mathscr{D}(\mathscr{C})$ is compact if the functor $\operatorname{Hom}_{\mathscr{D}(\mathscr{C})}(E, \cdot)$ commutes with infinite direct sums. The derived category $\mathscr{D}(\mathscr{C})$ is said to be spanned by a set of objects if for all nonzero objects $F$ of $\mathscr{D}(\mathscr{C})$, there exists an object $E$ in that set such that $\operatorname{Hom}_{\mathscr{D}(\mathscr{C})}(E, F) \neq 0$.

Theorem 1.3 [Rickard 1989, 6.4]. Suppose that the objects $E_{1}, \ldots, E_{n}$ are compact objects that span $\mathscr{D}(\mathscr{C})$, and that for all $i$ and $j$, we have $\operatorname{Ext}_{\mathscr{C}}^{k}\left(E_{i}, E_{j}\right)=0$ for all $k \neq 0$. Then $R F$ is an equivalence of triangulated categories.

Remark 1.4. Rickard's theorem further states that the equivalence $R F$ restricts to an equivalence of the full subcategories of compact objects (which are triangulated). If $\mathscr{b}=\mathrm{QCoh}(V)$ for an algebraic variety $V$ over $\mathfrak{k}$, then the compact objects of $\mathscr{D}(\mathscr{C})$ are those which are locally quasi-isomorphic to bounded complexes of locally free sheaves of finite rank. If $V$ is smooth, this is simply the class of all complexes quasi-isomorphic to a bounded complex of coherent sheaves. Thus, the 
full subcategory of compact objects is equivalent to $\mathscr{D}^{b}(\mathrm{Coh}(V))$, and the connection to the categories appearing in the introduction is apparent.

We will be interested in the case in which $\mathscr{C}=\mathrm{QCoh}(V)$ is the category of quasicoherent sheaves on a (not necessarily smooth) algebraic variety $V$ over $\mathfrak{k}$. In order to endow $\operatorname{End}_{V}\left(\oplus E_{i}\right)^{\text {op }}$ with an appropriate grading from which to construct a Bondal quiver, we need some extra structure on $V$ and extra conditions on the sheaves $E_{1}, \ldots, E_{n}$.

Definition 1.5. A variety $V$ equipped with an action of the multiplicative group $\mathbb{G}_{m}$ is called nearly projective if it is projective over its affinization $V_{0}=\operatorname{Spec} \Gamma\left(\mathcal{O}_{V}\right)$, the $\mathbb{G}_{m}$ action on $V_{0}$ has a unique fixed point, and $\mathbb{G}_{m}$ retracts $V_{0}$ to that fixed point. Algebraically, this means that we may write $V=\operatorname{Proj} R$ for an $\mathbb{N} \times \mathbb{Z}$-graded ring $R$ with $R_{0, i}=0$ for $i<0$ and $R_{0,0} \cong \mathfrak{k}$. Here the $\mathbb{N}$-grading is used to construct Proj, and the $\mathbb{Z}$-grading gives the $\mathbb{G}_{m}$ action on $V$.

Example 1.6. Any projective variety $V$ is nearly projective with respect to the trivial $\mathbb{G}_{m}$ action.

Example 1.7. Suppose that $X$ is projective with an ample line bundle $L^{-1}$, and let $V$ be the total space of $L$. Then $V$ is nearly projective with respect to the scaling action of $\mathbb{G}_{m}$ along the fibers.

Definition 1.8. Let $V$ be nearly projective, and let $E_{1}, \ldots, E_{n}$ be $\mathbb{G}_{m}$-equivariant vector bundles on $V$. We call this collection decent if $\operatorname{End}\left(E_{i}\right) \cong \Gamma\left(\mathcal{O}_{V}\right)$ for all $i$, $\mathbb{G}_{m}$ acts on the vector space $\operatorname{Hom}\left(E_{i}, E_{j}\right)$ with nonnegative weights for all pairs $i, j$, and it acts with positive weights if $j<i$.

Let $A=\operatorname{End}_{V}\left(\oplus E_{i}\right)^{\text {op }}$, and write

$$
A=\bigoplus_{\substack{1 \leq i, j \leq n, r \in \mathbb{Z}}} A_{i j}^{r},
$$

where $A_{i j}^{r}$ is the $r$-eigenspace of $\operatorname{Hom}\left(E_{i}, E_{j}\right)$ with respect to the action of $\mathbb{G}_{m}$. We define a grading on $A$ by assigning degree $j-i+n r$ to $A_{i j}^{r}$. The following proposition says that this grading has all of the properties required to define the Bondal quiver $\mathbf{Q}$ of the collection $\left\{E_{1}, \ldots, E_{n}\right\}$.

Proposition 1.9. If $V$ is nearly projective and $E_{1}, \ldots, E_{n}$ is decent, then this grading is nonnegative, the graded pieces are finite-dimensional, and the degree zero part is spanned by the idempotents $\left\{e_{1}, \ldots, e_{n}\right\}$.

Proof. The nonnegativity follows immediately from decency of $E_{1}, \ldots, E_{n}$. To establish the finite dimensionality of the graded pieces, it is sufficient to show that $A_{i j}^{r}$ is finite-dimensional for all $i, j, r$. Let $\pi_{0}: V \rightarrow V_{0}$ be the natural projection. Then $A_{i j}^{r}$ is equal to the $r$-eigenspace of sections of the sheaf $\left(\pi_{0}\right)_{*} \operatorname{Hom}\left(E_{i}, E_{j}\right)$ 
on $V_{0}$. Let us write $V=\operatorname{Proj} R$, and let $R_{0}$ be the degree zero piece with respect to the $\mathbb{N}$-grading. Then $V_{0}=\operatorname{Spec} R_{0}$, and the $\mathbb{G}_{m}$ action on $V_{0}$ induces an $\mathbb{N}$-grading on $R_{0}$ with degree zero piece $R_{0,0}$ equal to $\mathfrak{k}$. A $\mathbb{G}_{m}$-equivariant coherent sheaf on $V_{0}$ corresponds to a finitely generated graded $R_{0}$-module, and $A_{i j}^{r}$ is canonically isomorphic the degree $r$ part, which must be finite-dimensional.

The degree zero part of $A$ is equal to the direct sum $\bigoplus_{i} A_{i i}^{0}$. Since our collection is decent, $A_{i i}=\operatorname{End}_{V}\left(E_{i}\right)^{\mathrm{op}}$ is the free $R_{0}$-module of rank one generated in degree zero by a single class, namely $e_{i}$.

Definition 1.10. For any $\mathfrak{k}$-linear abelian category $\mathscr{C}$, an object $E$ in $\mathscr{C}$ is called exceptional if $\operatorname{End}_{\mathscr{C}}(E) \cong \mathfrak{k}$ and $E x t_{\mathscr{C}}^{k}(E, E)=0$ for $k \neq 0$. A collection $E_{1}, \ldots, E_{n}$ is called exceptional if each $E_{i}$ is exceptional and $\operatorname{Ext}_{\mathscr{b}}^{\bullet}\left(E_{i}, E_{j}\right)=0$ for all $i>j$. An exceptional collection is called full if it spans $\mathscr{D}(\mathscr{C})$, and strong if $\operatorname{Ext}_{\mathscr{C}}^{k}\left(E_{i}, E_{j}\right)=0$ for all $k \neq 0$ and all $i, j$.

Example 1.11. Let $V$ be an irreducible projective variety equipped with the trivial $\mathbb{G}_{m}$ action, and let $E_{1}, \ldots, E_{n}$ be a full, strong, exceptional collection of vector bundles on $V$ equipped with the trivial $\mathbb{G}_{m}$ action. Then the collection is decent, the Bondal quiver makes sense, and the hypotheses of Theorem 1.3 are satisfied. Such collections are known to exist on projective spaces of arbitrary dimension [Běllinson 1978], and on all odd-dimensional, smooth, quadric hypersurfaces [Kapranov 1986]. They are conjectured to exist on complete flag varieties of semisimple groups [Kuznetsov 2006, 1.2]. King [1997] shows that they exist on all smooth, Fano, toric surfaces, and Craw and Smith [2007] extend this result to smooth, Fano, toric 3-folds. Costa and Miró-Roig [2004] have found more toric examples in arbitrary dimension. King [1997, 9.3 and 9.4] conjectured that such a full, strong, exceptional collection exists on every smooth, projective toric variety, and (more generally) on any variety that may be obtained as a GIT quotient of a vector space by a linear action of a reductive group, provided that a polarization is chosen for which the notions of stability and semistability coincide. Kawamata [2006] showed that every smooth, projective toric variety admits a full exceptional collection, but not necessarily a strong one. Hille and Perling [2006] have recently constructed a toric counterexample to King's conjecture, but the question of how common such collections are is still wide open.

Example 1.12. Let $E_{1}, \ldots, E_{n}$ be a full, strong, exceptional collection of vector bundles on a smooth, projective variety $X$, and let $L$ be a line bundle on $X$ such that $L^{-1}$ is ample. We extend our collection infinitely in both directions via the formula

$$
E_{i-n}=E_{i} \otimes L \quad \text { for all } i \in \mathbb{Z}
$$


Such an infinite collection will be called a spiral with respect to L. A spiral will be called simple if it satisfies the equation

$$
\operatorname{Ext}_{X}^{k}\left(E_{i}, E_{j}\right)=0 \quad \text { for all } k \neq 0 \text { and } i \leq j .
$$

A simple spiral with respect to the canonical bundle with $n=\operatorname{dim} X+1$ will be called a simple helix. This notion will become important in Remark 2.9 and Section 4. ${ }^{1}$

Suppose that $E_{1}, \ldots, E_{n}$ generate a simple spiral with respect to $L$. Let $V$ denote the total space of $L$, and let $\pi: V \rightarrow X$ be the projection; $V$ is nearly projective by Example 1.7. For any pair $i, j$, we have

$$
\operatorname{Hom}_{V}\left(\pi^{*} E_{i}, \pi^{*} E_{j}\right) \cong \operatorname{Hom}_{X}\left(E_{i}, \pi_{*} \pi^{*} E_{j}\right) \cong \bigoplus_{r \geq 0} \operatorname{Hom}_{X}\left(E_{i}, E_{j} \otimes L^{-r}\right),
$$

where $r$ is the eigenvalue for the action of $\mathbb{G}_{m}$. Thus the collection $\pi^{*} E_{1}, \ldots, \pi^{*} E_{n}$ is decent, and the Bondal quiver is well defined.

For any $\mathscr{F} \in \mathscr{D}(\mathrm{QCoh}(V))$, we have

$$
\operatorname{Ext}_{V}^{\bullet}\left(\oplus \pi^{*} E_{i}, \mathscr{F}\right)=\operatorname{Ext}_{X}^{\bullet}\left(\oplus E_{i}, \pi_{*} \mathscr{F}\right),
$$

which is trivial if and only if $\mathscr{F}=0$. Hence $\pi^{*} E_{1}, \ldots, \pi^{*} E_{n} \operatorname{span} \mathscr{D}(\mathrm{QCoh}(V))$. The condition (1) ensures that the bundles $\pi^{*} E_{1}, \ldots, \pi^{*} E_{n}$ have no higher Ext groups between them, so Theorem 1.3 applies to this collection.

We henceforth assume that $V$ is nearly projective and that $E_{1}, \ldots, E_{n}$ is a decent collection of vector bundles with Bondal quiver $\mathbf{Q}$. We do not assume that the derived functor $R F$ is an equivalence, unless we say so explicitly. Let $\alpha_{i}=\operatorname{rank} E_{i}$ be a vector of natural numbers, and let $\mathscr{R e p}(\mathbf{Q})$ denote the substack of the moduli stack of representations of $\mathbf{Q}$ for which the vector space associated to the node $i$ has dimension $\alpha_{i}$. Over each point in the variety $V$, the fiber of the vector bundle $\bigoplus E_{i}^{\vee}$ is naturally a left-module over the algebra $\operatorname{End}_{V}\left(\bigoplus E_{i}\right)^{\mathrm{op}} \cong P(\mathbf{Q})$. Thus, $V$ parametrizes a family of representations, and we have a tautological map

$$
T: V \rightarrow \mathscr{R e p}_{\alpha}(\mathbf{Q})
$$

On the level of points, we have $T(p)=F\left(\mathcal{O}_{p}\right)$, where $\mathcal{O}_{p}$ is the structure sheaf of the point $p \in V$.

Theorem 1.13. If $V$ is smooth and $R F$ is an equivalence of derived categories, then $T$ is injective and induces an isomorphism on tangent spaces.

\footnotetext{
${ }^{1}$ Our definition of a simple helix agrees with that of [Bridgeland 2005, Section 3]; the same structure is called a geometric helix in [Bondal and Polishchuk 1993, Section 1]. "Helix" by itself is used inconsistently in the literature, and we will never use it. The term "spiral" is our own.
} 
Proof. The facts that each $E_{i}$ is a vector bundle and $O_{p}$ has zero-dimensional support tell us that all of the higher right-derived functors of $F$ vanish on $\mathrm{O}_{p}$. Hence $R F\left(\mathrm{O}_{p}\right)=F\left(\mathrm{O}_{p}\right)$ is an honest representation rather than a complex of representations. The injectivity of $T$ then follows from the fact that the objects $\left\{O_{p} \mid p \in V\right\}$ are all nonisomorphic in $\mathscr{D} \operatorname{QCoh}(V) \cong \mathscr{D} \operatorname{Rep}(\mathbf{Q})$ and, hence, in the full subcategory $\operatorname{Rep}(\mathbf{Q})$.

To see that $T$ induces an isomorphism on tangent spaces, we note that we have a sequence of isomorphisms

$$
\begin{aligned}
T_{p} V \cong \operatorname{Ext}_{V}^{1}\left(\mathscr{O}_{p}, \mathscr{O}_{p}\right) & \cong \operatorname{Hom}_{\mathscr{Q} Q \operatorname{Coh}(V)}\left(\mathscr{O}_{p}, \mathscr{O}_{p}[1]\right) \\
& \cong \operatorname{Hom}_{\mathscr{D} \operatorname{Rep}(\mathbf{Q})}\left(R F\left(\mathscr{O}_{p}\right), R F\left(\mathscr{O}_{p}\right)[1]\right) \\
& \cong \operatorname{Ext}_{\operatorname{Rep} \mathbf{Q}}^{1}\left(F\left(\mathscr{O}_{p}\right), F\left(\mathscr{O}_{p}\right)\right) \\
& \cong T_{T(p)} \mathscr{R e p}_{\alpha} \mathbf{Q}
\end{aligned}
$$

Let $\mathbb{D}=\operatorname{Spec} \mathfrak{k}[\epsilon] /\left\langle\epsilon^{2}\right\rangle$. Then tangent vectors to $V$ and $\mathscr{R e p}_{\alpha} \mathbf{Q}$ are represented respectively by maps $e: \mathbb{D} \rightarrow V$ and families of quiver representations over $\mathbb{D}$, and the differential of $T$ sends $e \in T_{p} V$ to $\bigoplus e^{*} E_{i}^{\vee} \in T_{T(p)} \mathscr{R e p}_{\alpha} \mathbf{Q}$. It remains only to show that this map coincides with the isomorphism of (2).

Consider the Cartesian square

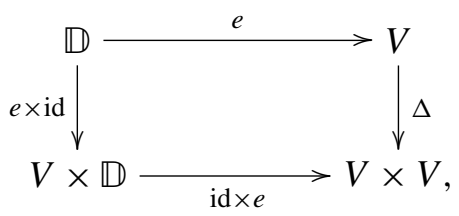

and let $\pi: V \times \mathbb{D} \rightarrow \mathbb{D}$ and $\rho: V \times \mathbb{D} \rightarrow V$ denote the projections. An element of $T_{p} V \cong \operatorname{Ext}_{V}^{1}\left(O_{p}, O_{p}\right)$ may be regarded as a family of coherent sheaves on $V$ parameterized by $\mathbb{D}$, or, equivalently, as a coherent sheaf on $V \times \mathbb{D}$. In these terms, the element represented by $e: \mathbb{D} \rightarrow V$ may be identified with the coherent sheaf

$$
(\mathrm{id} \times e)^{*} \Delta_{*} \mathrm{O}_{V}=(e \times \mathrm{id})_{*} e^{*} \mathscr{O}_{V} .
$$

Then the family of quiver representations obtained by applying $F$ is

$$
\begin{aligned}
\pi_{*} \mathcal{H} \mathrm{om}_{V \times \mathbb{D}}\left(\bigoplus \rho^{*} E_{i},(e \times \mathrm{id})_{*} e^{*} \mathscr{O}_{V}\right) & =\pi_{*}(e \times \mathrm{id})_{*} \mathcal{H}_{\mathrm{om}_{\mathbb{D}}}\left(\bigoplus e^{*} E_{i}, e^{*} \mathscr{O}_{V}\right) \\
& =\mathcal{H}_{\mathrm{om}}\left(\bigoplus e^{*} E_{i}, e^{*} \mathfrak{O}_{V}\right) \\
& =\bigoplus e^{*} E_{i}^{\vee},
\end{aligned}
$$

which is precisely the tangent vector to $\mathscr{R} \mathrm{ep}_{\alpha} \mathbf{Q}$ obtained by applying the differential of $T$ to $e$. Thus the isomorphism of (2) is indeed the one induced by $T$. 


\section{Semistable representations}

As in Section 1, let $E_{1}, \ldots, E_{n}$ be a decent collection of $\mathbb{G}_{m}$-equivariant vector bundles on a nearly projective algebraic variety $V$ over $\mathfrak{k}$, and let $\alpha_{i}=\operatorname{rank} E_{i}$. Let $R_{\alpha}(\mathbf{Q})$ be the set of representations of $\mathbf{Q}$ in which the node $i$ is mapped to a fixed coordinate vector space $\mathfrak{k}^{\alpha_{i}}$. This set has the structure of an affine algebraic variety over $\mathfrak{k}$.

Example 2.1. Let $V=\mathbb{P}^{2}$, and let $E_{1}=\mathcal{O}, E_{2}=\mathcal{O}(1)$, and $E_{3}=\mathcal{O}(2)$. The following picture represents the category $\mathscr{C}(\mathbf{Q})$, with each arrow labeled by the vector space of morphisms between the corresponding objects.

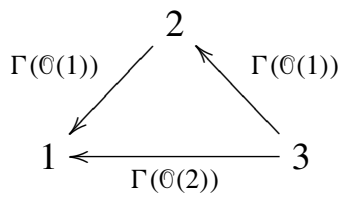

The quiver itself consists of three arrows from 2 to 1 and three arrows from 3 to 2 , representing bases for the vector space $\Gamma(\mathcal{O}(1))$. There are no arrows from 3 to 1 , because the multiplication map

$$
\psi: \Gamma(\mathcal{O}(1)) \otimes \Gamma(\mathcal{O}(1)) \rightarrow \Gamma(\mathcal{O}(2))
$$

is surjective. An element of $R_{\alpha}(\mathbf{Q})$ consists of a pair of vectors $a_{12}, a_{23} \in \Gamma(\mathbb{O}(1))^{\vee}$ such that $a_{12} \otimes a_{23}$ lies in the image of $\psi^{\vee}$. In concrete terms, this means that $a_{12}$ and $a_{23}$ must be proportional.

Let $G=\prod_{i} \mathrm{GL}\left(\alpha_{i}\right) / \mathbb{G}_{m}^{\text {diag }}$. This group acts naturally on $R_{\alpha}(\mathbf{Q})$ by the formula

$$
\left(g_{1}, \ldots, g_{n}\right) \cdot\left(a_{i j}\right)=\left(g_{i} a_{i j} g_{j}^{-1}\right),
$$

and two representations are isomorphic if and only if they lie in the same $G$-orbit. Any representation of $\mathbf{Q}$ in which all nodes are mapped to vector spaces of the given dimension is isomorphic to an element of $R_{\alpha}(\mathbf{Q})$; this is just the statement that all finite-dimensional vector spaces of a given dimension are isomorphic. It follows that the stack $\mathscr{R e p}_{\alpha}(\mathbf{Q})$, considered in the previous section, is represented by the quotient $\left[R_{\alpha}(\mathbf{Q}) / G\right]$.

Let $\chi=\left(\chi_{1}, \ldots, \chi_{n}\right)$ be an ordered $n$-tuple of integers satisfying $\sum \chi_{i} \alpha_{i}=0$. We may interpret $\chi$ as a multiplicative character of the group $G$ by the formula $g \mapsto \operatorname{det}\left(g_{1}\right)^{\chi_{1}} \cdots \operatorname{det}\left(g_{n}\right)^{\chi_{n}}$. Let $M_{\chi}=R_{\alpha}(\mathbf{Q}) / /_{\chi} G$ be the semiprojective GIT quotient of $R_{\alpha}(\mathbf{Q})$ by $G$ with respect to the character $\chi$. This quotient has two equivalent interpretations, which we describe below. ${ }^{2}$

\footnotetext{
${ }^{2}$ Geometric invariant theory was originally developed by Mumford [1994], but what we need is summarized in the short survey [Proudfoot 2005].
} 
Let $B$ be the affine coordinate ring of $R_{\alpha}(\mathbf{Q})$. The action of $G$ on $R_{\alpha}(\mathbf{Q})$ induces an action on $B$. For any character $\theta$ of $G$, let $B(\theta)$ be the $\theta$-eigenspace of $B$, and let $B_{\chi}=\bigoplus_{r \geq 0} B(r \chi)$. The GIT quotient $M_{\chi}$ is defined as Proj $B_{\chi}$. This definition makes it clear that $M_{\chi}$ is a variety equipped with an ample line bundle, making $M_{\chi}$ projective over its affinization $M_{0}=\operatorname{Spec} B^{G}$.

An element $a$ of $R_{\alpha}(\mathbf{Q})$ is called $\chi$-semistable if there is a function $f \in B(r \chi)$ for some $r>0$ such that $f(a) \neq 0$. The locus of semistable points is an open subset of $R_{\alpha}(\mathbf{Q})$ and will be denoted $R_{\alpha}(\mathbf{Q})^{\chi-s s}$. Such a representation is called $\chi$-stable if its stabilizer is finite and its $G$-orbit is closed in $R_{\alpha}(\mathbf{Q})^{\chi-s s}$. The locus of stable points is an open subset of $R_{\alpha}(\mathbf{Q})^{\chi-s s}$ and will be denoted $R_{\alpha}(\mathbf{Q})^{\chi-s t}$. Two semistable representations are called $S$-equivalent if the closures of their $G$ orbits intersect in $R_{\alpha}(\mathbf{Q})^{\chi-s s}$. There is a surjective map from $R_{\alpha}(\mathbf{Q})^{\chi-s s}$ to $M_{\chi}$ whose fibers are precisely the S-equivalence classes, so $M_{\chi}$ may be thought of as the moduli space of semistable representations of $\mathbf{Q}$ with dimension vector $\alpha$, up to S-equivalence.

Recall the tautological map $T: V \rightarrow \mathscr{R e p}_{\alpha} \mathbf{Q}$. The variety $M_{\chi}$ is a quotient of an open substack of $\mathscr{R e p}_{\alpha} \mathbf{Q}$, so $T$ induces a rational map $T_{\chi}: V \rightarrow M_{\chi}$. If $T_{\chi}$ is in fact regular, meaning that every tautological representation $T(p)$ is $\chi$-semistable, we will say that the character $\chi$ is $\operatorname{good}$. If in fact $T(p)$ is $\chi$-stable for all $p$, we will say that $\chi$ is great.

As a first step to analyzing the map $T_{\chi}$ for various values of $\chi$, we must consider the case where $\chi=0$. In this case, $T \chi$ factors through the affinization map

$$
\pi_{0}: V \rightarrow V_{0}:=\operatorname{Spec} \Gamma\left(\mathscr{O}_{V}\right)
$$

via the map

$$
\varphi_{0}: V_{0} \rightarrow M_{0}=\operatorname{Spec} B^{G}=\operatorname{Spec} \Gamma\left(\mathscr{O}_{\mathscr{R e p}_{\alpha}} \mathbf{Q}\right)
$$

obtained by pulling back global functions from $\mathscr{R e p}_{\alpha} \mathbf{Q}$ to $V$. We note that every element of $R_{\alpha}(\mathbf{Q})$ is semistable with respect to the trivial character, so $\chi=0$ is always good.

Proposition 2.2. The map $\varphi_{0}: V_{0} \rightarrow M_{0}$ is a closed embedding.

Proof. This is equivalent to the statement that $T^{*}: \Gamma\left(\mathcal{O}_{\mathscr{R e p}} \mathbf{Q}\right) \rightarrow \Gamma\left(\mathscr{O}_{V}\right)$ is surjective. Choose any node $i$. The isomorphism $\Gamma\left(\mathscr{O}_{V}\right) \cong \operatorname{End}\left(E_{i}\right)$ coming from the decency of the collection allows us to identify the ring of global functions on $V$ with the algebra of loops in $\mathbf{Q}$ based at $i$. For any function $f \in \Gamma\left(\mathscr{O}_{V}\right)$, let $s_{i}(f)$ be the $G$-invariant function on $R_{\alpha}(\mathbf{Q})$ taking a representation to $1 / \alpha_{i}$ times the trace of the endomorphism obtained by going around the loop corresponding to $f$. Then $T^{*} s_{i}(f)=f$. 
Remark 2.3. If $\alpha_{i}=1$, then $s_{i}$ is a homomorphism and induces a map $\sigma_{i}: M_{0} \rightarrow V_{0}$ of which $\varphi_{0}$ is a section. In general, however, $s_{i}$ fails to be an isomorphism because trace is not multiplicative.

For any character $\chi$, consider the line bundle

$$
E_{\chi}=\operatorname{det}\left(E_{1}\right)^{\otimes \chi_{1}} \otimes \cdots \otimes \operatorname{det}\left(E_{n}\right)^{\otimes \chi_{n}} .
$$

Theorem 2.4. Suppose that $V$ is smooth, $R F$ induces an equivalence of derived categories, and $\chi$ is great. Then $T_{\chi}$ identifies $V$ with a connected component of $M_{\chi}$, and $E_{\chi}$ with the line bundle induced by the GIT construction.

Proof. Since $\chi$ is great, $T_{\chi}$ maps $V$ to the stable locus of $M_{\chi}$, which is isomorphic to an open substack of $\mathscr{R e} p_{\alpha} \mathbf{Q}$. Theorem 1.13 tells us that $T_{\chi}$ is injective on points and induces an isomorphism on tangent spaces. Since $V$ is smooth, this implies that $T_{\chi}$ is an isomorphism onto a Zariski open subset of $M_{\chi}$.

Since $V$ is nearly projective, it is projective over its affinization $V_{0}$, and $M_{\chi}$ is projective over $M_{0}$. Since $T_{\chi}: V \rightarrow M_{\chi}$ covers the closed immersion $T_{0}: V_{0} \rightarrow M_{0}$, its image must be closed. Thus $T_{\chi}$ is an isomorphism onto a connected component of $M_{\chi}$.

To prove the final statement, we note that the character $\chi$ defines an equivariant structure on the trivial line bundle on $R_{\alpha}(\mathbf{Q})$, which descends to a nontrivial line bundle $L_{\chi}$ on the stack quotient $\left[R_{\alpha}(\mathbf{Q}) / G\right] \cong \mathscr{R e p}_{\alpha} \mathbf{Q}$. The GIT line bundle on $M_{\chi}$ is obtained by restricting $L_{\chi}$ from $\mathscr{R e p}_{\alpha} \mathbf{Q}$, so it will suffice to show that $T^{*} L_{\chi}=E_{\chi}$.

Let $\tilde{\mathscr{G}}$ be the principal $\prod_{i} \mathrm{GL}\left(\alpha_{i}\right)$-bundle on $V$ associated to the vector bundle $E=\bigoplus_{i} E_{i}$, and let $\mathscr{G}$ be the principle $G$-bundle obtained by dividing $\tilde{\mathscr{G}}$ by $\mathbb{G}_{m}^{\mathrm{diag}}$. Then we have a pullback diagram of principal $G$-bundles

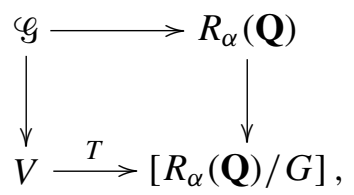

and the line bundles $E_{\chi}$ and $L_{\chi}$ are the line bundles associated to these principle bundles via the one-dimensional representation of $G$ given by the character $\chi$. The statement follows.

Remark 2.5. More generally, the rational map $T_{\chi}: V \rightarrow M_{\chi}$ factors through the rational map $\pi_{\chi}: V \rightarrow V_{\chi}$ via a third rational map $\varphi_{\chi}: V_{\chi} \rightarrow M_{\chi}$. The maps $\pi_{\chi}$ and $\varphi_{\chi}$ will both be regular if and only if $\chi$ is good.

Remark 2.6. Craw and Smith [2007] obtain a result similar to Theorem 2.4, but with different hypotheses. The most important differences are that they restrict to collections of line bundles and that they assume that $V$ is toric. In exchange, 
they are able to substantially weaken the assumption that $F$ is an equivalence of categories.

The remainder of this section will be devoted to giving sufficient criteria for $\chi$ to be good or great in the case where each $E_{i}$ is a line bundle. There is a simple description of stability and semistability of quiver representations due to King [1994, Section 3], which we summarize in the special case of representations that are one-dimensional at each node. For any subset $S \subseteq\{1, \ldots, n\}$, let $\chi_{S}=$ $\sum_{i \in S} \chi_{i}$. We define the support of a representation of $\mathbf{Q}$ to be the set of nodes that map to nonzero vector spaces. A representation $a \in R_{\alpha}(\mathbf{Q})$ has a subrepresentation with support $S$ if and only if $a_{i j}=0$ for all $i \in S^{c}$ and $j \in S$. King tells us that $a$ is $\chi$-semistable if and only if $\chi_{S} \leq 0$ for all supports $S$ of subrepresentations of $a$, and $a$ is $\chi$-stable if equality is obtained only by the trivial representation and $a$ itself.

Let $\left\{m_{i j}\right\}$ be a collection of nonnegative integers, and define $\chi$ by the formula

$$
\chi=\sum_{i, j} m_{i j} \cdot(0, \ldots, 0,-1,0, \ldots, 0,1,0, \ldots, 0),
$$

where -1 appears in the $i$-th spot, and 1 in the $j$-th spot. Equivalently, we put

$$
\chi_{\ell}=\sum_{i=1}^{n} m_{i \ell}-\sum_{j=1}^{n} m_{\ell j} \quad \text { for all } \ell \leq n .
$$

Proposition 2.7. If $\mathscr{H o m}\left(E_{i}, E_{j}\right)$ is generated by global sections for all $i$ and $j$ such that $m_{i j} \neq 0$, then $\chi$ is good.

Proof. Let $S$ be the support of a subrepresentation of $T(p)$ for some $p \in V$. We need to show that $\chi_{S} \leq 0$, where

$$
\chi_{S}=\sum_{\ell \in S} \chi_{\ell}=\sum_{\ell \in S} \sum_{i=1}^{n} m_{i \ell}-\sum_{j=1}^{n} \sum_{\ell \in S} m_{\ell j} .
$$

The condition that $\mathscr{H o m}\left(E_{i}, E_{j}\right)$ is generated by global sections says exactly that $T(p)_{i j}$ (the part of the quiver representation that records the homomorphisms from $\left.E_{i}^{\vee}\right|_{p}$ at node $i$ to $\left.E_{j}^{\vee}\right|_{p}$ at node $j$ ) is nonzero for all $p \in V$. Thus if $m_{i j} \neq 0$ and $j \in S$, then $i$ must be in $S$ as well. This tells us that every term that appears with a plus sign above also appears with a minus sign, and therefore that $\chi_{S} \leq 0$.

We will say that $\left\{m_{i j}\right\}$ is sufficient if the following two conditions are satisfied:

(i) $\operatorname{Hom}\left(E_{i}, E_{j}\right)$ is generated by global sections for all $i, j$ such that $m_{i j} \neq 0$.

(ii) It is possible to get from any one vertex of $Q$ to any other by traveling forward along paths from $j$ to $i$ such that $\mathscr{H o m}\left(E_{i}, E_{j}\right)$ is generated by global sections, and backward along paths from $j$ to $i$ such that $m_{i j} \neq 0$. 
Proposition 2.8. If $\left\{m_{i j}\right\}$ is sufficient, then $\chi$ is great.

Proof. Let $S$ be the support of a nonzero subrepresentation of $T(p)$ for some $p \in V$, and suppose that $\chi_{S}=0$. We need to show that $S=\{1, \ldots, n\}$. If $j \in S$ and $\operatorname{Hom}\left(E_{i}, E_{j}\right)$ is generated by global sections, then $T(p)_{i j} \neq 0$, and therefore $i \in S$. If $i \in S$ and $m_{i j} \neq 0$, then $-m_{i j}$ is a summand in Equation (3). Since every positive summand is canceled by a negative one and $\chi_{S}=0$, the term $m_{i j}$ must appear as well, hence $j \in S$. In this manner, we can conclude that the set $S$ is closed under the two operations described in condition (ii) above. Since $S$ is nonempty, it must contain all of $\{1, \ldots, n\}$.

Remark 2.9. Suppose that $E_{1}, \ldots, E_{n}$ generate a simple helix on a projective variety $X$, as in Example 1.12. Bondal and Polishchuk [1993, 2.5] show that for all $1 \leq i \leq n-1$, the object $R_{E_{i+1}} E_{i} \in \mathscr{D} \operatorname{Coh}(X)$ defined by the exact triangle

$$
E_{i+1} \rightarrow \operatorname{Hom}\left(E_{i}, E_{i+1}\right)^{\vee} \otimes E_{i+1} \rightarrow R_{E_{i+1}} E_{i}
$$

is pure; in other words, it lies in the abelian subcategory $\operatorname{Coh}(X)$. This is equivalent to the statement that the first map in the triangle is injective, or that its dual is surjective. This in turn is the statement that $\operatorname{Hom}\left(E_{i}, E_{i+1}\right)$ is generated by global sections, and therefore so is $\mathcal{H o m}\left(E_{i}, E_{j}\right)$ for all $i \leq j$. Furthermore, they prove that the endomorphism algebra $\operatorname{End}\left(\bigoplus E_{i}\right)$ is multiplicatively generated by elements of the vector spaces $\operatorname{Hom}\left(E_{i}, E_{i+1}\right)$, which implies that 1 is the unique source of $\mathbf{Q}$ and $n$ is the unique sink. In this case, therefore, $\left\{m_{i j}\right\}$ is sufficient if and only if $m_{1 n}>0$.

Remark 2.9 gives us many examples of characters $\chi$ that satisfy the hypotheses of Theorem 2.4 when our collection generates a simple helix. This will apply to the collection $\mathbb{O}, \mathcal{O}(1) \ldots, \mathcal{O}(n)$ on $\mathbb{P}^{n}$ [Beylinson 1978], as well as to collections on odd-dimensional quadrics [Kapranov 1986]. We conclude this section with an example in which Remark 2.9 does not apply, but Propositions 2.7 and 2.8 do.

Example 2.10. Let $V$ be the Hirzebruch surface $\mathbb{F}_{1}$, the blow-up of $\mathbb{P}^{2}$ at a single point. Consider the collection $E_{1}=\mathcal{O}, E_{2}=\mathcal{O}(D), E_{3}=\mathcal{O}(H)$, and $E_{4}=\mathcal{O}(2 H)$, where $H$ is the proper transform of a hyperplane class in $\mathbb{P}^{2}$ and $D$ is the exceptional divisor. This collection is full, strong, and exceptional, and has the following Bondal quiver, where the integers above the arrows indicate the number of distinct arrows between the two nodes (unlabeled arrows occur with multiplicity one). There are nontrivial relations among paths from 4 to 1 and among maps from 4 to 2 .

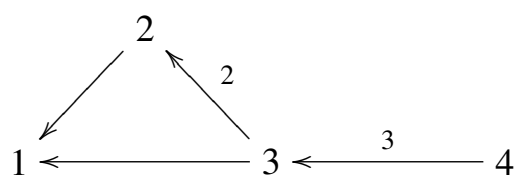


The only nonzero path in $\mathbf{Q}$ corresponding to a $\mathscr{H}$ om sheaf that is not generated by global sections is the arrow from 2 to 1 . Let $m_{14}=m_{23}=1$, and set all other $m_{i j}$ equal to zero. Then $\left\{m_{i j}\right\}$ is sufficient, so $\chi=(-1,-1,1,1)$ is great. Then Theorem 2.4 tells us that $M_{\chi}$ has a connected component that is isomorphic to $V$ in its projective embedding given by the anticanonical bundle $E_{\chi}=\mathrm{O}(3 H-D)$.

\section{D-branes at the tip of a cone}

In this section we continue to assume that $\alpha_{i}=1$ for all $i$. Suppose that a collection $E_{1}, \ldots, E_{n}$ of line bundles generates a simple spiral with respect to another line bundle $L$ on a smooth projective variety $X$, as in Example 1.12. Let $V$ denote the total space of $L$, and let $\mathbf{Q}$ be the Bondal quiver for the decent collection $\pi^{*} E_{1}, \ldots, \pi^{*} E_{n}$ on $V$. Theorem 3.1 and Corollary 3.2 generalize the main result of [Bergman and Proudfoot 2006].

Theorem 3.1. The map $\varphi_{0}$ is generically an isomorphism. More precisely, there exists a dense open subset $U \subseteq V_{0}$ such that $\left.\varphi_{0}\right|_{U}$ is an isomorphism onto its image, which is open in $M_{0}$.

Proof. We first observe that for all $i$ and $j$, there exist elements

$$
\begin{aligned}
& p_{i j} \in \operatorname{Hom}_{V}\left(\pi^{*} E_{i}, \pi^{*} E_{j}\right) \cong \bigoplus_{r \geq 0} \operatorname{Hom}_{X}\left(E_{i}, E_{j} \otimes L^{-r}\right), \\
& q_{i j} \in \operatorname{Hom}_{V}\left(\pi^{*} E_{j}, \pi^{*} E_{i}\right) \cong \bigoplus_{r \geq 0} \operatorname{Hom}_{X}\left(E_{j}, E_{i} \otimes L^{-r}\right)
\end{aligned}
$$

with nonzero product $\beta_{i j}=q_{i j} \cdot p_{i j} \in \operatorname{End}_{V}\left(\pi^{*} E_{i}\right) \cong \Gamma\left(\mathscr{O}_{V}\right)$. This follows from the ampleness of $L^{-1}$, which ensures that the vector spaces on the right will be nonzero for large values of $r$.

Recall from Remark 2.3 that for each node $i$ we have a homomorphism $s_{i}$ : $\Gamma\left(\mathscr{O}_{V}\right) \rightarrow \Gamma\left(\mathcal{O}_{M_{0}}\right)$ inducing a map $\sigma_{i}: M_{0} \rightarrow V_{0}$ such that $\sigma_{i} \circ \varphi_{0}=\mathrm{id}_{V}$. Le Bruyn and Procesi [1990, Theorem 1] show that the images of $s_{1}, \ldots, s_{n}$ generate $\Gamma\left(\mathcal{O}_{M_{0}}\right)$. Furthermore, for any element $r \in \operatorname{Hom}\left(E_{j}, E_{j}\right)$, we have

$$
s_{i}\left(\beta_{i j}\right) \cdot s_{j}(r)=s_{i}\left(q_{i j} \cdot p_{i j}\right) \cdot s_{j}(r)=s_{i}\left(p_{i j} \cdot r \cdot q_{i j}\right) .
$$

This means that $s_{i}$ becomes surjective after inverting the elements $s_{j}\left(\beta_{i j}\right) \in \Gamma\left(O_{M_{0}}\right)$ for all $j$. Geometrically, this tells us that there exists a dense open set $U_{i}$ of $V_{0}$ (the set on which $0 \neq \varphi_{0}^{*} s_{j}\left(\beta_{i j}\right)=\beta_{i j}$ for all $j$ ) over which $\sigma_{i}$ is an isomorphism. Since $\varphi_{0}$ is a section of $\sigma_{i}$, we are done.

Corollary 3.2. The map $\varphi_{0}$ identifies $V_{0}$ with the canonical reduced subvariety of an irreducible component of $M_{0}$. In particular, if $M_{0}$ is reduced, then $\varphi_{0}$ is an isomorphism onto an irreducible component. 
Proof. This follows from Proposition 2.2 and Theorem 3.1.

Remark 3.3. When $X$ is a Fano surface and $L=K_{X}$, this example has an interpretation in string theory. The quiver variety $M_{0}$ is the moduli space of vacua for ground states of open strings ending on a D-brane at the tip of the cone $V_{0}$; see for example [Bergman and Proudfoot 2006]. Considerations from topological string theory imply that one component of this moduli space should correspond to deformations of the D-brane away from the tip, and this component is the one picked out by $V_{0}$.

Remark 3.4. Suppose that a character $\chi$ is good for the collection $E_{1}, \ldots, E_{n}$ on $X$, in the sense of Section 2. Then $\chi$ is also good for the collection $\pi^{*} E_{1}, \ldots, \pi^{*} E_{n}$ on $V$. (This is because the quiver for the latter collection is obtained by adding arrows to the quiver for the original collection, thus making it easier for representations to be semistable.) The quiver variety $M_{\chi}$ for the collection on $V$ is projective over $M_{0}$, and the component into which $V$ maps by the tautological map is a partial resolution of $V_{0} \subseteq M_{0}$. It's easy to check that this partial resolution is an isomorphism away from the tip of the cone, and that the fiber over the tip is isomorphic to the variety $X_{\chi}$ introduced in Section 2.

Example 3.5. Let $X=\mathbb{P}^{1} \times \mathbb{P}^{1}$ with the exceptional collection $E_{1}=0, E_{2}=$ $\mathcal{O}(0,1), E_{3}=\mathcal{O}(1,0)$, and $E_{4}=\mathscr{O}(1,1)$. This collection generates a simple spiral

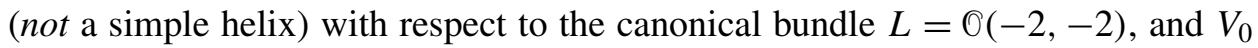
is isomorphic to the quotient of the conifold $\{x y-z w=0\} \subseteq \mathbb{C}^{4}$ by the diagonal action of $\mathbb{Z} / 2$. For a more detailed exposition of this example, see [Bergman and Proudfoot 2006, Section 4].

\section{A canonical projective quotient}

Suppose that $E_{1}, \ldots, E_{n}$ are line bundles that generate a simple helix on a smooth projective variety $X$. In other words, we are in the situation of Section 3 with $L=K_{X}$ the canonical bundle and $n=\operatorname{dim} X+1$. Let $\mathbf{Q}$ be the Bondal quiver associated to the collection $E_{1}, \ldots, E_{n}$ on $X$, and $\mathbf{Q}^{\prime}$ the Bondal quiver associated to the collection $\pi^{*} E_{1}, \ldots, \pi^{*} E_{n}$ on the total space $V$ of $K_{X}$. Then the underlying quiver $Q$ has arrows from $i+1$ to $i$ given by a basis for the vector space $\operatorname{Hom}_{X}\left(E_{i}, E_{i+1}\right)$, and no arrows between nonadjacent vertices [Bridgeland 2005, Section 4]. Similarly, Bridgeland shows that $Q^{\prime}$ is obtained from $Q$ by adding arrows from 1 to $n$ given by a basis for $\operatorname{Hom}_{X}\left(E_{n}, E_{1} \otimes K_{X}^{-1}\right)$.

By Theorem 1.3 and Example 1.12, the derived functors

$$
R F: \mathscr{D} \mathrm{QCoh}(X) \rightarrow \mathscr{D} \operatorname{Rep}(\mathbf{Q}) \quad \text { and } \quad R F^{\prime}: \mathscr{D} \operatorname{QCoh}(V) \rightarrow \mathscr{D} \operatorname{Rep}\left(\mathbf{Q}^{\prime}\right)
$$


are both equivalences of categories. Then by Theorem 1.13 and the fact that $X$ and $V$ are smooth, the tautological maps

$$
T: X \rightarrow \mathscr{R e p}_{\alpha} \mathbf{Q} \text { and } T^{\prime}: V \rightarrow \mathscr{R e p}_{\alpha} \mathbf{Q}^{\prime}
$$

are open immersions, and therefore the loci of points in $R_{\alpha}(\mathbf{Q})$ and $R_{\alpha}\left(\mathbf{Q}^{\prime}\right)$ lying over the images of these maps are open. Let $R_{\alpha}(\mathbf{Q})_{\text {taut }}$ and $R_{\alpha}\left(\mathbf{Q}^{\prime}\right)_{\text {taut }}$ be the closures of these loci; since $X$ is irreducible, $R_{\alpha}(\mathbf{Q})_{\text {taut }}$ and $R_{\alpha}\left(\mathbf{Q}^{\prime}\right)_{\text {taut }}$ are irreducible components of $R_{\alpha}(\mathbf{Q})$ and $R_{\alpha}\left(\mathbf{Q}^{\prime}\right)$. We will introduce resolutions $\tilde{R}_{\alpha}(\mathbf{Q})$ and $\tilde{R}_{\alpha}\left(\mathbf{Q}^{\prime}\right)$ of $R_{\alpha}(\mathbf{Q})_{\text {taut }}$ and $R_{\alpha}\left(\mathbf{Q}^{\prime}\right)_{\text {taut }}$, respectively. We will then show that, under certain hypotheses, $\tilde{R}_{\alpha}\left(\mathbf{Q}^{\prime}\right)$ has the structure of a $G$-equivariant line bundle over $\tilde{R}_{\alpha}(\mathbf{Q})$, and that the GIT quotient of $\tilde{R}_{\alpha}(\mathbf{Q})$ with respect to this line bundle is equal to $X$ in its anticanonical projective embedding. We thus recover $X$ as a GIT quotient of a smooth variety by $G$, without having to make any choice of character.

Since $\alpha_{i}=1$ for all $i$, the affine variety $R_{\alpha}(\mathbf{Q})$ admits a particularly simple explicit description. We have

$$
\begin{array}{ll}
R_{\alpha}(\mathbf{Q})=\left\{\left(a_{i j}\right) \in \prod_{i, j} \operatorname{Hom}\left(E_{i}, E_{j}\right)^{\vee} \mid \psi_{i j k}^{\vee}\left(a_{i k}\right)=a_{i j} \otimes a_{j k}\right. & \text { for all } i, j, k \\
& \text { and } \left.a_{i i}\left(\operatorname{id}_{E_{i}}\right)=1\right\},
\end{array}
$$

where

$$
\psi_{i j k}: \operatorname{Hom}_{X}\left(E_{i}, E_{j}\right) \otimes \operatorname{Hom}_{X}\left(E_{j}, E_{k}\right) \rightarrow \operatorname{Hom}_{X}\left(E_{i}, E_{k}\right)
$$

is the natural composition map and

$$
\psi_{i j k}^{\vee}: \operatorname{Hom}_{X}\left(E_{i}, E_{k}\right)^{\vee} \rightarrow \operatorname{Hom}_{X}\left(E_{i}, E_{j}\right)^{\vee} \otimes \operatorname{Hom}_{X}\left(E_{j}, E_{j}\right)^{\vee}
$$

is its dual. Let

where

$$
\begin{aligned}
\tilde{R}_{\alpha}(\mathbf{Q}) & =\left\{(a, \ell) \mid a_{i j} \in \ell_{i j} \text { and } \Psi_{i j k}^{\vee}\left(\ell_{i k}\right)=\ell_{i j} \otimes \ell_{j k}\right\} \\
& \subseteq R_{\alpha}(\mathbf{Q}) \times \prod_{i<j} \mathbb{P}\left(\operatorname{Hom}_{X}\left(E_{i}, E_{j}\right)\right),
\end{aligned}
$$

$$
\Psi_{i j k}^{\vee}: \mathbb{P}\left(\operatorname{Hom}_{X}\left(E_{i}, E_{k}\right)\right) \rightarrow \mathbb{P}\left(\operatorname{Hom}_{X}\left(E_{i}, E_{j}\right)\right) \otimes \mathbb{P}\left(\operatorname{Hom}_{X}\left(E_{j}, E_{k}\right)\right)
$$

is the projectivization of $\psi_{i j k}^{\vee}$. Note that for $\Psi_{i j k}^{\vee}$ to be well defined, we need $\psi_{i j k}^{\vee}$ to be injective or equivalently $\psi_{i j k}$ to be surjective. This, however, is guaranteed by the fact that $P(\mathbf{Q})$ is generated by arrows between adjacent nodes. Note that an element of $R_{\alpha}(\mathbf{Q})$ is determined by the coordinates $a_{i i+1}$ for all $i<n$, and an element of $\tilde{R}_{\alpha}(\mathbf{Q})$ is determined by these data along with the lines $\ell_{i+1}$, but for notational purposes it is still useful to keep track of $a_{i j}$ and $\ell_{i j}$ for all $i<j$. 
The space $\tilde{R}_{\alpha}\left(\mathbf{Q}^{\prime}\right)$ will be defined in a similar manner, but the fact that $\mathbf{Q}^{\prime}$ has loops makes the definition slightly more delicate. Recall that, for all $i, j$, we have

$$
\operatorname{Hom}_{V}\left(\pi^{*} E_{i}, \pi^{*} E_{j}\right) \cong \bigoplus_{r \geq 0} \operatorname{Hom}_{X}\left(E_{i}, E_{j} \otimes K_{X}^{-r}\right) .
$$

An element $a \in R_{\alpha}\left(\mathbf{Q}^{\prime}\right)$ may be regarded as a collection $\left(a_{i j}^{r}\right)$ of elements $a_{i j}^{r} \in$ $\operatorname{Hom}_{X}\left(E_{i}, E_{j} \otimes K_{X}^{-r}\right)^{\vee}$ that satisfies the equations

$$
\left(\psi_{i j k}^{r s}\right)^{\vee}\left(a_{i k}^{r+s}\right)=a_{i j}^{r} \otimes a_{j k}^{s},
$$

where $\psi_{i j k}^{r s}$ is the restriction of $\psi_{i j k}$ to the $(r, s)$-graded piece of the product

$$
\operatorname{Hom}_{V}\left(\pi^{*} E_{i}, \pi^{*} E_{j}\right) \times \operatorname{Hom}_{V}\left(\pi^{*} E_{j}, \pi^{*} E_{k}\right) .
$$

We then define

$$
\begin{aligned}
\tilde{R}_{\alpha}\left(\mathbf{Q}^{\prime}\right) & =\left\{(a, \ell) \mid a_{i j}^{r} \in \ell_{i j}^{r} \text { and }\left(\Psi_{i j k}^{r s}\right)^{\vee}\left(\ell_{i k}^{r+s}\right)=\ell_{i j}^{r} \otimes \ell_{j k}^{s}\right\} \\
& \subseteq R_{\alpha}\left(\mathbf{Q}^{\prime}\right)_{\text {taut }} \times \prod_{i, j, r} \mathbb{P}\left(\operatorname{Hom}_{X}\left(E_{i}, E_{j} \otimes K_{X}^{-r}\right)\right),
\end{aligned}
$$

where $\left(\Psi_{i j k}^{r s}\right)^{\vee}$ is the projectivization of $\left(\psi_{i j k}^{r s}\right)^{\vee}$. Once again, these maps are well defined because the maps $\psi_{i j k}^{r s}$ are surjective, which follows from Bridgeland's description of $\mathbf{Q}^{\prime}$. As in the case of $\mathbf{Q}$, an element of $\tilde{R}_{\alpha}\left(\mathbf{Q}^{\prime}\right)$ is completely determined by the data

$$
\begin{aligned}
& a_{i+1}^{0} \in \ell_{i i+1}^{0} \subseteq \operatorname{Hom}_{V}\left(\pi^{*} E_{i}, \pi^{*} E_{i+1}\right)_{0}^{\vee} \cong \operatorname{Hom}_{X}\left(E_{i}, E_{i+1}\right)^{\vee} \text { for all } i<n \\
& \text { and } a_{n 1}^{1} \in \ell_{n 1}^{1} \subseteq \operatorname{Hom}_{V}\left(\pi^{*} E_{n}, \pi^{*} E_{1}\right)_{1}^{\vee} \cong \operatorname{Hom}_{X}\left(E_{n}, E_{1} \otimes K_{X}^{-1}\right)^{\vee},
\end{aligned}
$$

subject to certain relations.

Consider the $G$-equivariant projection from $\tilde{R}_{\alpha}\left(\mathbf{Q}^{\prime}\right)$ to $\tilde{R}_{\alpha}(\mathbf{Q})$ given by remembering only the degree zero parts $a^{0}$ and $\ell^{0}$.

Proposition 4.1. Suppose that there exist nonnegative integers $\left\{m_{i j}\right\}$ such that

$$
\operatorname{Hom}_{X}\left(E_{n}, E_{1} \otimes K_{X}^{-1}\right) \cong \bigotimes_{i, j} \operatorname{Hom}_{X}\left(E_{i}, E_{j}\right)^{\otimes m_{i j}} .
$$

Then the projection from $\tilde{R}_{\alpha}\left(\mathbf{Q}^{\prime}\right)$ to $\tilde{R}_{\alpha}(\mathbf{Q})$ has the structure of an equivariant line bundle.

Proof. Given an element $\left(a^{0}, \ell^{0}\right) \in \tilde{R}_{\alpha}(\mathbf{Q})$, we will show that the degree one line

$$
\ell_{n 1}^{1} \subseteq \mathbb{P}\left(\operatorname{Hom}_{X}\left(E_{n}, E_{1} \otimes K_{X}^{-1}\right)\right)
$$

of a preimage is uniquely determined, and that any point $a_{n 1}^{1} \in \ell_{n 1}^{1}$ extends $\left(a^{0}, \ell^{0}\right)$ to an element of $\tilde{R}_{\alpha}\left(\mathbf{Q}^{\prime}\right)$. To see $\ell_{n 1}^{1}$ is uniquely determined, let $m=\max _{i, j}\left\{m_{i j}\right\}$. 
By composing maps of the form $\Psi_{i+1 i+2}$ as we wrap $m$ times around the quiver $\mathbf{Q}^{\prime}$, we find that

$$
\ell_{n 1}^{m} \mapsto\left(\ell_{12}^{0}\right)^{\otimes m} \otimes \ldots \otimes\left(\ell_{n-1 n}^{0}\right)^{\otimes m} \otimes\left(\ell_{n 1}^{1}\right)^{\otimes m} .
$$

The right side of the line above contains $\left(\ell_{12}^{0}\right)^{\otimes m_{12}} \otimes \cdots \otimes\left(\ell_{n-1 n}^{0}\right)^{\otimes m_{n-1 n}} \otimes \ell_{n 1}^{1}$ as a factor. The lines $\left(\ell_{12}^{0}\right)^{\otimes m_{12}} \otimes \ldots \otimes\left(\ell_{n-1 n}^{0}\right)^{\otimes m_{n-1 n}}$ and $\ell_{n 1}^{1}$ are both contained in $\mathbb{P}\left(\operatorname{Hom}_{X}\left(E_{n}, E_{1} \otimes K_{X}^{-1}\right)\right.$, and the symmetries of the compositions of the maps $\Psi_{i j k}$ imply that anything in their image is invariant under the interchanging of these factors. Thus $\ell_{n 1}^{1}$ must be equal to $\left(\ell_{12}^{0}\right)^{\otimes m_{12}} \otimes \ldots \otimes\left(\ell_{n-1 n}^{0}\right)^{\otimes m_{n-1 n}}$.

The defining equations for $R_{\alpha}\left(\mathbf{Q}^{\prime}\right)$ are linear in $a_{n 1}^{0}$, and therefore to see that they are satisfied by every element $a_{n 1}^{1} \in \ell_{n 1}^{1}$, it will suffice to find one element of $\tilde{R}_{\alpha}\left(\mathbf{Q}^{\prime}\right)$ lying over $\left(a^{0}, \ell^{0}\right)$ in which $a_{n 1}^{1}$ is nonzero. Suppose that the image of $a^{0}$ in the stack $\mathscr{R e p} \alpha \mathbf{Q}$ is equal to $T(p)$ for some $p \in X$. In other words, $a^{0}$ is obtained from $p$ by choosing an isomorphism $\left.E_{i}^{\vee}\right|_{p} \cong \mathfrak{k}$ for each $i$. Let $q \in V$ be a nonzero element of the fiber of $K_{X}$ at $p$. Our choices of trivializations of the vector spaces $\left.E_{i}^{\vee}\right|_{p}$ induce trivializations of the pullbacks $\left.\pi^{*} E_{i}^{\vee}\right|_{q}$, and thus $T^{\prime}(q) \in \mathscr{R e p}_{\alpha} \mathbf{Q}^{\prime}$ lifts naturally to an element of $R_{\alpha}\left(\mathbf{Q}^{\prime}\right)$ extending $a^{0}$. In Remark 2.9, we observed that the helix condition ensures that $\mathcal{H} \mathrm{om}_{X}\left(E_{i}, E_{i+1}\right)$ is generated by global sections for all $i$. This observation applies equally well when $i=n$, so $\operatorname{Hom}_{X}\left(E_{n}, E_{1} \otimes K_{X}^{-1}\right)$ is also generated by global sections. It follows that $T^{\prime}(q)$ lifts further to a unique element of $\tilde{R}_{\alpha}\left(\mathbf{Q}^{\prime}\right)$ extending $\left(a^{0}, \ell^{0}\right) \in \tilde{R}_{\alpha}(\mathbf{Q})$, and that $a_{n 1}^{1} \neq 0$.

We have now shown that the fibers of the map from $\tilde{R}_{\alpha}\left(\mathbf{Q}^{\prime}\right)$ to $\tilde{R}_{\alpha}(\mathbf{Q})$ are vector spaces of dimension at most one, and that the dimension is equal to one over those elements $\left(a^{0}, \ell^{0}\right)$ such that $a^{0}$ lies over the image of $T$. But this is a dense open condition, and the dimension of the fiber of an algebraic map is an upper semicontinuous function. Hence the dimension of the fiber must always be exactly one.

Example 4.2. Consider the data of Example 2.1, in which $X=\mathbb{P}^{2}$ and $R_{\alpha}(\mathbf{Q})$ is the variety of pairs of proportional vectors in the three-dimensional vector space $\Gamma(\mathcal{O}(1))^{\vee}$. The quiver $\mathbf{Q}^{\prime}$ is obtained by adding arrows from node 1 to node 3 indexed by the vector space $\operatorname{Hom}(\mathcal{O}(2), \mathcal{O}(3)) \cong \Gamma(\mathcal{O}(1))$, and the relations tell us that an element of $R_{\alpha}\left(\mathbf{Q}^{\prime}\right)$ is a triple of proportional vectors in $\Gamma(\mathcal{O}(1))^{\vee}$. The projection from $R_{\alpha}\left(\mathbf{Q}^{\prime}\right)$ to $R_{\alpha}(\mathbf{Q})$ has fibers which are generically lines, but the fiber over zero is a vector space of dimension 3. After blowing up the origin of $\Gamma(\mathcal{O}(1))^{\vee}$, we obtain

$$
\begin{aligned}
\tilde{R}_{\alpha}(\mathbf{Q}) & =\left\{a_{12}, a_{23} \in \ell \subseteq \Gamma(\mathbb{O}(1))^{\vee}\right\}, \\
\tilde{R}_{\alpha}\left(\mathbf{Q}^{\prime}\right) & =\left\{a_{12}^{0}, a_{23}^{0}, a_{31}^{1} \in \ell \subseteq \Gamma(\mathbb{O}(1))^{\vee}\right\},
\end{aligned}
$$

and the fibers of the induced projection are all lines. 
If the conclusion of Proposition 4.1 holds, let $\mathscr{L}$ be the dual of the corresponding line bundle.

Theorem 4.3. The GIT quotient of $\tilde{R}_{\alpha}(\mathbf{Q})$ with respect to the polarization $\mathscr{L}$ is isomorphic to $X$, with line bundle $K_{X}^{-1}$.

Proof. Let $\chi_{m}$ be the character associated to the collection $\left\{m_{i j}\right\}$ as in Section 2, and let $\chi=\chi_{m}+(-1,0, \ldots, 0,1)$, so that $E_{\chi} \cong K_{X}^{-1}$. The fact that $\mathscr{H}_{\mathrm{om}}\left(E_{i}, E_{i+1}\right)$ is generated by global sections ensures that the natural projection from $\tilde{R}_{\alpha}(\mathbf{Q})$ to $R_{\alpha}(\mathbf{Q})_{\text {taut }}$ is an isomorphism over $R_{\alpha}(\mathbf{Q})_{\text {taut }}^{\chi-s t}$, and this isomorphism identifies $\mathscr{L}$ with the restriction of the trivial line bundle on $R_{\alpha}(\mathbf{Q})$ twisted by the character $\chi$. Thus $\tilde{R}_{\alpha}(\mathbf{Q}) / / \mathscr{L} G$ is isomorphic to the tautological component of $R_{\alpha}(\mathbf{Q}) / / \chi G=$ $M_{\chi}$, which is isomorphic to $X$ in its anticanonical embedding by Theorem 2.4, Proposition 2.8, and Remark 2.9.

Remark 4.4. We note that the collection $\left\{m_{i j}\right\}$ satisfying the hypotheses of Proposition 4.1 may not be unique. In Example 2.1, we may take $m_{12}=1$ and $m_{23}=0$, or vice versa; these choices give us two different characters $\chi$ which define the same stability condition on $R_{\alpha}(\mathbf{Q})$. Theorem 4.3 , on the other hand, requires no choices.

Remark 4.5. Since $\tilde{R}_{\alpha}\left(\mathbf{Q}^{\prime}\right)$ is projective over $R_{\alpha}\left(\mathbf{Q}^{\prime}\right)_{\text {taut }}$, they have the same ring of global functions. This tells us that

$$
\operatorname{Spec} \Gamma\left(\mathscr{O}_{\tilde{R}_{\alpha}\left(\mathbf{Q}^{\prime}\right)}^{G}\right)=\operatorname{Spec} \Gamma\left(\mathcal{O}_{R_{\alpha}\left(\mathbf{Q}^{\prime}\right)_{\text {taut }}}^{G}\right)=R_{\alpha}\left(\mathbf{Q}^{\prime}\right)_{\text {taut }} / / 0 G,
$$

the underlying reduced variety of which isomorphic to $V_{0}$ by Theorem 3.1. Recall that $V_{0}$ is obtained from $V$ by collapsing the zero section of $K_{X}$ to a point. The GIT quotient $\tilde{R}_{\alpha}(\mathbf{Q}) / / \mathscr{L} G$ is isomorphic to $\operatorname{Proj} 0_{\tilde{R}_{\alpha}\left(\mathbf{Q}^{\prime}\right)}^{G}$, which is obtained from

$$
\operatorname{Spec} \Gamma\left(\mathcal{O}_{\tilde{R}_{\alpha}\left(\mathbf{Q}^{\prime}\right)}^{G}\right) \cong V_{0}
$$

by throwing away the tip of the cone and dividing by the natural action of $\mathbb{G}_{m}$. This tells us that any nonreduced structure of $R_{\alpha}\left(\mathbf{Q}^{\prime}\right)_{\text {taut }} / /{ }_{0} G$ must be concentrated at the tip of the cone. If we had known this fact a priori, then it would have constituted an alternative proof of Theorem 4.3.

Remark 4.6. We have used the hypothesis that the collection $E_{1}, \ldots, E_{n}$ generates a simple helix throughout this section, but we remark that in some examples, our methods may be applied to a simple spiral as well. Consider, for example, the collection $\mathcal{O}, \mathcal{O}(1,0), \mathcal{O}(1,1), \mathcal{O}(2,1)$ on $\mathbb{P}^{1} \times \mathbb{P}^{1}$, extended to a simple spiral by the canonical bundle $O(-2,-2)$. (Note that this is not the same collection that we used in Example 3.5.) In this case, $Q$ has arrows only between adjacent nodes with all composition maps surjective, and $Q^{\prime}$ is obtained from $Q$ by adding arrows from 
the first node to the last. Thus we may define $\tilde{R}_{\alpha}(\mathbf{Q})$ and $\tilde{R}_{\alpha}\left(\mathbf{Q}^{\prime}\right)$ exactly as we do in the helix case, and Theorem 4.3 will still hold.

\section{Acknowledgments}

The authors are grateful to David Ben-Zvi, Brian Conrad, Deepak Khosla, and Gregory Smith for invaluable discussions. We are especially grateful to Alastairs Craw and King for their detailed comments and suggestions.

\section{References}

[Aspinwall and Donagi 1998] P. S. Aspinwall and R. Y. Donagi, "The heterotic string, the tangent bundle and derived categories", Adv. Theor. Math. Phys. 2:5 (1998), 1041-1074. MR 2000b:81116 Zbl 0961.81091

[Bey̌linson 1978] A. A. Beйlinson, "Coherent sheaves on $\mathbf{P}^{n}$ and problems in linear algebra", Funktsional. Anal. i Prilozhen. 12:3 (1978), 68-69. In Russian. MR 80c:14010b

[Bergman and Proudfoot 2006] A. Bergman and N. Proudfoot, "Moduli spaces for D-branes at the tip of a cone", J. High Energy Phys. 3 (2006), 073, 9 pp. MR 2007d:14025

[Bondal and Polishchuk 1993] A. I. Bondal and A. E. Polishchuk, "Homological properties of associative algebras: the method of helices", Izv. Ross. Akad. Nauk Ser. Mat. 57:2 (1993), 3-50. In Russian; translated in Russian Acad. Sci. Izv. Math. 42:2 (1994), 219-260. MR 94m:16011 Zbl 0847.16010

[Bridgeland 2005] T. Bridgeland, "t-structures on some local Calabi-Yau varieties", J. Algebra 289:2 (2005), 453-483. MR 2006a:14067 Zbl 1069.14044

[Costa and Miró-Roig 2004] L. Costa and R. M. Miró-Roig, "Tilting sheaves on toric varieties", Math. Z. 248:4 (2004), 849-865. MR 2005m:14023 Zbl 1071.14022

[Craw and Smith 2007] A. Craw and G. Smith, "Projective toric varieties as fine moduli spaces of quiver representations", 2007. To appear in Am. J. Math. arXiv math.AG/0608183v2

[Douglas 2001] M. R. Douglas, "D-branes, categories and $\mathcal{N}=1$ supersymmetry", J. Math. Phys. 42:7 (2001), 2818-2843. Strings, branes, and M-theory. MR 2003b:81158 Zbl 1036.81027

[Gabriel 1962] P. Gabriel, "Des catégories abéliennes", Bull. Soc. Math. France 90 (1962), 323-448. MR 38 \#1144 Zbl 0201.35602

[Hille and Perling 2006] L. Hille and M. Perling, "A counterexample to King's conjecture", Compos. Math. 142:6 (2006), 1507-1521. MR 2007h:14074 Zbl 1108.14040

[Kapranov 1986] M. M. Kapranov, "Derived category of coherent bundles on a quadric", Funktsional. Anal. i Prilozhen. 20:2 (1986), 67. In Russian; translated in Functional Anal. Appl. 20:2 (1986), 141-142. MR 88a:14014 Zbl 0607.18004

[Kawamata 2006] Y. Kawamata, "Derived categories of toric varieties", Michigan Math. J. 54:3 (2006), 517-535. MR 2008d:14079

[King 1994] A. D. King, "Moduli of representations of finite-dimensional algebras", Quart. J. Math. Oxford Ser. (2) 45:180 (1994), 515-530. MR 96a:16009 Zbl 0837.16005

[King 1997] A. King, “Tilting bundles on some rational surfaces”, Preprint, 1997, Available at http:// www.maths.bath.ac.uk/ masadk/papers/tilt.ps.

[Kontsevich 1995] M. Kontsevich, "Homological algebra of mirror symmetry", pp. 120-139 in Proceedings of the International Congress of Mathematicians (Zürich, 1994), vol. 1, edited by S. D. Chatterji, Birkhäuser, Basel, 1995. MR 97f:32040 Zbl 0846.53021 
[Kuznetsov 2006] A. Kuznetsov, "Exceptional collections for grassmannians of isotropic lines", 2006. To appear in Proc. London Math. Soc. arXiv math.AG/0512013v2

[Le Bruyn and Procesi 1990] L. Le Bruyn and C. Procesi, "Semisimple representations of quivers", Trans. Amer. Math. Soc. 317:2 (1990), 585-598. MR 90e:16048 Zbl 0693.16018

[Mumford et al. 1994] D. Mumford, J. Fogarty, and F. Kirwan, Geometric invariant theory, 3rd ed., Ergebnisse der Mathematik und ihrer Grenzgebiete (2) 34, Springer, Berlin, 1994. MR 95m:14012

[Proudfoot 2005] N. Proudfoot, "Geometric invariant theory and projective toric varieties", pp. 161167 in Snowbird lectures in algebraic geometry, Contemp. Math. 388, Amer. Math. Soc., Providence, RI, 2005. MR 2006j:14065 arXiv math/0502366v2

[Rickard 1989] J. Rickard, "Morita theory for derived categories", J. London Math. Soc. (2) 39:3 (1989), 436-456. MR 91b:18012 Zbl 0642.16034

[Segal 2007] E. Segal, "The A-infinity deformation theory of a point and the derived categories of local Calabi-Yaus”, Preprint, 2007. arXiv math.AG/0702539v2

[Sharpe 1999] E. Sharpe, "D-branes, derived categories, and Grothendieck groups", Nuclear Phys. B 561:3 (1999), 433-450. MR 2001k:14024 Zbl 1028.81519

Received February 9, 2008.

\author{
AARON BERGMAN \\ PHYSICS DEPARTMENT \\ TEXAS A\&M UNIVERSITY \\ COLlEge Station, TX 77843-4242 \\ UNITED STATES \\ abergman@physics.tamu.edu \\ Nicholas J. Proudfoot \\ DEPARTMENT OF MATHEMATICS \\ 1222 UNIVERSITY OF OREGON \\ EUGENE, OR 97403 \\ UNITED STATES \\ njp@uoregon.edu
}

